Journal of Surgical Sciences (2013) Vol. 16 (2) : 52-53

(C) 2012 Society of Surgeons of Bangladesh

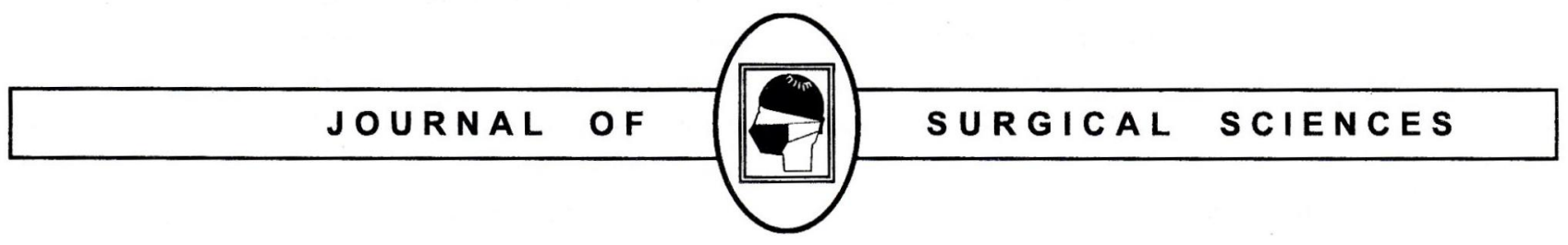

\title{
Editorial
}

\section{MOLECULAR BASIS OF GASTRIC CARCINOMA - A CHALLENGE FOR THE FUTURE DAYS}

Some of the earliest observations in cancer biology as well as recent advances in molecular analyses contribute to our knowledge about the multistep process of gastric carcinogenesis.

The gastrointestinal tract has rapid epithelial turnover and exposure to injury by infections and dietary toxins. These conditions create very high cancer prevalence. Intestinalization of gastric units, which is called "IM"; phenotypic antralization of fundic units, which is called "spasmolytic polypeptide-expressing metaplasia (SPEM)"; and the development directly from the stem/ progenitor cell zone, are three pathways that have been described for gastric carcinogenesis ${ }^{1}$.

Neoplasia can follow cellular metaplasia due to chronic inflammation, injury and repair. This is the most documented process for gastric cancer ${ }^{2}$. An acceptable concept is that there are two corner-stones with regard to this process. Firstly, the initial observation of Rudolf Virchow in 1863 about leucocytes in neoplastic tissues and the connection between inflammation and cancer; secondly, about 15 years ago, researchers' evidence about the relationship between stomach cancer and infection by Helicobacter pylori (H. pylori) (isolated by Drs. Marshall and Warren in 1984. Also, we must note that Epstein-Barr virus has been detected in stomach tissues in approximately $10 \%$ of gastric carcinoma cases $^{3}$.

Beginning with some of the earliest observations in cancer biology, it appears that chronic inflammation stimulates tumor development and plays a critical role in initiating, sustaining and advancing tumor growth. Direct effect of the viral pathogens on neoplastic transformation of epithelial cells has been shown; however, it is also evident that not all inflammation is tumorigenic. It can be suggested that either the tumor alters the immune response by reactive oxygen species and cytokines or chronic inflammation plays a primary role in transforming tissue cells (especially mentioned in "stem cell theory") into tumor cells. In the acute phase of inflammation, the release of endogenous reactive oxygen and nitrogen species $\left(\mathrm{O}_{2}\right.$, $\mathrm{H}_{2} \mathrm{O}_{2}, \mathrm{NO}, \mathrm{OH}, \mathrm{ONOO}^{-}, \mathrm{HOCl}$ ) from such innate immune cells as macrophages and leukocytes plays an important role in the elimination of pathogens. However, when present chronically, this can induce DNA damage in proliferating cells. In addition, it is also possible for other bacteria to colonize the stomach and additionally trigger carcinogenesis by gastric atrophy (result of chronic inflammation) which represents a loss of gastric glands and associated lower acidity of gastric juice. Hypoacidity associated with $\mathrm{H}$. pylori infection induces gastric mucosal atrophy to advance multistage carcinogenesis in the stomach. Interleukin (IL)-1 $\beta$, IL-6, IL-8, tumor necrosis factor- $\alpha$ (TNF- $\alpha$ ) and interferon- $\gamma$ (IFN- $\gamma$ ) are elevated in gastric mucosa with $H$. pylori infection. Gastrin is upregulated and acid secretion from parietal cells is inhibited mainly due to pro-inflammatory cytokines IL-1 $1 \beta$ and TNF- $\alpha$. TNF- $\alpha$ and IL- $1 \beta$ are essential in the initiation of chronic inflammation. Recent works have shown that IL-1 $\beta$ overexpression, in the absence of Helicobacter infection, is sufficient to cause gastric cancer and it is one of the essential proinflammatory cytokines modulated during $H$. pylori infection that directs the mucosa toward atrophy, metaplasia, and neoplastic transformation ${ }^{4}$. Another important point that should 
be added is that $H$. pylori has been consistently associated with higher risk of gastric noncardia cancer. The inverse association of $\mathrm{H}$. pylori with gastric cardia cancer or esophageal adenocarcinoma has been shown in several studies, especially in Western populations. Furthermore, mast cells in particular play an important role in attracting inflammatory cells by releasing inflammatory mediators. Monocytes differentiate into macrophages, and become activated in response to local chemokine and cytokine interactions. Recently, direct evidence has also linked IL-6 to inflammation-mediated tumor initiation and proliferation in colon cancer. IL-6 can inhibit dendritic cell maturation and, together with the NF-êB-activating cytokines IL-1 and TNF, can promote tumor progression. Cytokines also affect cell death and cell cycle, pathways. TNF- $\alpha$ is produced mainly by macrophages. It is also produced by tumor cells. TNF$\alpha$ is associated with tissue destruction and plays a role in destroying tumor blood supply. However, if it is produced chronically, it can act as a tumor promoter by contributing to tissue remodeling and stromal development ${ }^{5}$. Nuclear factor (NF)-êB and STAT3 pathways have emerged as key regulators of the release of these pro-inflammatory cytokines, and important mediators of both tumor proliferation and persistence of chronic inflammation. The activation of these pathways results in further cytokine release ${ }^{6}$.

Although the subsequent pathways are different, chronic inflammation is the first step in both the intestinal and the diffuse type of gastric cancer. While the intestinal type has a sequence of multifocal atrophic gastritis, IM and dysplasia, which advances to carcinoma, the diffuse type tends to be primarily genetic in origin ${ }^{7}$. The progress from $\mathrm{IM}$ to gastric cancer has a wide range of molecular alterations affecting transcription factors, such as CDX1 and CDX2, telomerases, microsatellite instability, mutations of p53 protein, overexpression of COX-2, cyclin D2, and decreased expression of $\mathrm{p} 27$. The next step is gastric dysplasia. During the progression of normal tissue through the metaplasia-dysplasia sequence, there are mutations in genes including $p 53$, also loss of heterozygosity of the adenomatous polyposis coli gene, overexpression of the antiapoptotic gene $b c /-2$ and a mixture of polyploidy and aneuploidy.

Inflammation also plays an important role in the ability of tumor cells to invade and metastasize. The ability of epithelial tumor cells which metastasize to express specific chemokine receptors has been shown.
Paracrine secretion of pro-inflammatory cytokines (i.e., IL-1 $\beta$, IL-6, TNF- $\alpha$ ) and certain autocrine cytokine production support this process. During the later stages, additional mutations can be acquired, and this leads to the cancer cell gaining a further growth advantage and acquiring a more malignant phenotype ${ }^{8}$.

The origin of human gastric Cancer Stem Cells has yet to be elucidated, but data obtained from mouse model of Helicobacter-induced gastric cancer have implicated bone marrow-derived cells as a potential candidate. Further studies focusing on the identification and characterization of CSCs in gastric cancer may lead to novel diagnostic and therapeutic tools, dramatically improving the prognosis of gastric cancer patients.

\section{Md. Mizanur Rahman}

Department of Surgical Oncology, National Institute of Cancer Research and Hospital, Mohakhali, Dhaka.

\section{References:}

1. Fox JG, Wang TC. Inflammation, atrophy, and gastric cancer. J Clin Invest. 2007;117:60-69.

2. Gonda TA, Tu S, Wang TC. Chronic inflammation, the tumor microenvironment and carcinogenesis. Cell Cycle. 2009;8:2005-2013.

3. Takada K. Epstein-Barr virus and gastric carcinoma. Mol Pathol. 2000;53:255-261.

4. El-Omar EM. The importance of interleukin 1 beta in Helicobacter pylori associated disease. Gut. 2001;48:743-747.

5. El-Omar EM. The importance of interleukin 1 beta in Helicobacter pylori associated disease. Gut. 2001;48:743-747.

6. Rius J, Guma M, Schachtrup C, Akassoglou K, Zinkernagel AS, Nizet V, Johnson RS, Haddad GG, Karin M. NF-kappaB links innate immunity to the hypoxic response through transcriptional regulation of HIF-1alpha. Nature. 2008;453:807811.

7. Nardone G, Rocco A, Malfertheiner P. Review article: helicobacter pylori and molecular events in precancerous gastric lesions. Aliment Pharmacol Ther. 2004;20:261-270.

8. Weinberg RA. Mechanisms of malignant progression. Carcinogenesis. 2008;29:10921095 . 